\title{
ANALYTIC MODULI SPACES OF SIMPLE SHEAVES ON FAMILIES OF INTEGRAL CURVES
}

\author{
IGOR BURBAN AND BERND KREUSSLER
}

\begin{abstract}
We prove the existence of fine moduli spaces of simple coherent sheaves on families of irreducible curves. Our proof is based on the existence of a universal upper bound of the Castelnuovo-Mumford regularity of such sheaves, which we provide.
\end{abstract}

\section{INTRODUCTION}

In [10] and 99 a geometric associative (resp. classical) $r$-matrix was associated to any flat analytical family of integral curves of genus one. This way one can construct families of solutions to the associative (resp. classical) Yang-Baxter equation extending an earlier approach of [18].

Both constructions are based on the existence of a fine moduli space of simple vector bundles on an analytical family of integral curves of arithmetic genus one.

The result of the present article is more general. We prove the existence of a fine relative moduli space of simple sheaves on any flat family of integral projective curves of fixed arithmetic genus with a section. A coherent sheaf $\mathcal{F}$ on an integral projective curve $E$ over $\mathbb{C}$ is called simple if the natural linear map $\mathbb{C} \rightarrow \operatorname{Hom}_{E}(\mathcal{F}, \mathcal{F})$ is an isomorphism.

The exact assumptions are the following.

- Let $p: X \longrightarrow S$ be a flat projective morphism of complex spaces of relative dimension one and denote by $\breve{X}$ the smooth locus of $p$.

- Assume there exists a section $i: S \longrightarrow \breve{X}$ of $p$.

- Suppose that for all points $s \in S$ the fibre $X_{s}$ is a reduced and irreducible projective curve of fixed arithmetic genus $g$.

By $\mathrm{An}_{S}$ we denote the category of (not necessarily separated) complex spaces over $S$. If $f: T \rightarrow S$ is an object of this category, we obtain a cartesian diagram

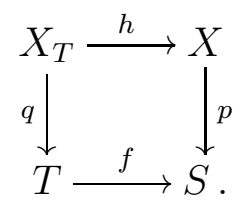

For each $t \in T$, we denote by $X_{t}$ the fibre of $q$ over $t$. If $\mathcal{F} \in \operatorname{Coh}\left(X_{T}\right)$, we denote by $\mathcal{F}_{t}$ the restriction of $\mathcal{F}$ to the fibre $X_{t}$. 
Given two coprime integers $n \geq 0$ and $d$, we define the functor $\underline{\mathrm{M}}_{X / S}^{(n, d)}: \mathrm{An}_{S} \rightarrow$ Sets as follows:

$$
\underline{\mathrm{M}}_{X / S}^{(n, d)}(T \stackrel{f}{\rightarrow} S)=\left\{\mathcal{F} \in \operatorname{Coh}\left(X_{T}\right) \mid \begin{array}{l}
\mathcal{F} \text { is } T \text {-flat, } \mathcal{F}_{t} \text { is simple } \\
\operatorname{rk}\left(\mathcal{F}_{t}\right)=n, \chi\left(\mathcal{F}_{t}\right)=d \quad \forall t \in T
\end{array}\right\} / \sim
$$

where $\mathcal{F}_{1} \sim \mathcal{F}_{2}$ if and only if there exists $\mathcal{L} \in \operatorname{Pic}(T)$ such that $\mathcal{F}_{1} \cong \mathcal{F}_{2} \otimes q^{*}(\mathcal{L})$. By $\operatorname{rk}\left(\mathcal{F}_{t}\right)$ we denote the rank of $\mathcal{F}_{t}$ at the generic point of the integral curve $X_{t}$.

Our main result, Theorem 4.1, states that this functor is representable by a complex space over $S$. For the proof we use a universal upper bound for the CastelnuovoMumford regularity of simple sheaves with fixed $(n, d)$, provided in Section 3 .

Our result may seem to look well known and familiar at the first glance. And indeed, in the category of schemes, the representability was shown in the early 1960s by Grothendieck [13] for $(n, d)=(1, d)$. For general $(n, d)$ Altman and Kleiman [1] showed at the end of the 1970s representability as an algebraic space, but they considered the sheafification in the étale topology of the functor defined above.

Similarly, in the analytic category, localised (or sheafified) functors were shown to be representable for $S$ a point by Norton [17] in 1979, in the case $(n, d)=(1, d)$ for general $S$ by Bingener [7] in 1980 and in general by Kosarew and Okonek [16] in 1989. For our application, mentioned at the beginning, we indeed need the representability of the functor $\underline{\mathrm{M}}_{X / S}^{(n, d)}$ as defined above and not only of the sheafified version of it. Therefore, we need to prove that this functor is already a sheaf in the analytic topology; this is the main contribution we make in this paper.

\section{Preliminaries}

2.1. Local invariants. A one-dimensional local Noetherian ring is Cohen-Macaulay if its maximal ideal contains a non-zero divisor. Let $(A, \mathfrak{m}, k)$ be a local Noetherian domain of dimension one. Then $A$ is Cohen-Macaulay, $\operatorname{Hom}_{A}(k, A)=0$ and $\operatorname{Ext}_{A}^{1}(k, A) \neq 0$. The number

$$
\mathrm{t}(A)=\operatorname{dim}_{k} \operatorname{Ext}_{A}^{1}(k, A)
$$

is called the type of $A$. The ring $A$ is Gorenstein iff $\mathrm{t}(A)=1$, see [8, Thm. 3.2.10]. Let $A \subset \widetilde{A} \subset Q(A)$ be the normalisation (integral closure) of $A$ in its field of fractions $Q(A)$. Throughout we assume that $A$ is the localisation of a $k$-algebra of finite type or $k=\mathbb{C}$ and $A$ is a local complex analytic algebra. Such rings are excellent and so by [11, Theorem 6.5], $\widetilde{A}$ is a finite $A$-module, which ensures that $\widetilde{A} / A$ has finite length. The $\delta$-invariant of $A$ is the length of the Artinian $A$-module $\widetilde{A} / A$, i.e.

$$
\delta(A)=\operatorname{dim}_{k}(\widetilde{A} / A) .
$$

Lemma 2.1. If $A$ is not regular, then $\mathrm{t}(A) \leq \delta(A)$. 
Proof. Applying $\operatorname{Hom}_{A}(\cdot, A)$ to the exact sequence $0 \rightarrow \mathfrak{m} \rightarrow A \rightarrow k \rightarrow 0$ yields the exact sequence of $A$-modules

$$
0 \longrightarrow A \longrightarrow \operatorname{Hom}_{A}(\mathfrak{m}, A) \longrightarrow \operatorname{Ext}_{A}^{1}(k, A) \longrightarrow 0 \text {. }
$$

We would like to compare it with the exact sequence $0 \rightarrow A \rightarrow \widetilde{A} \rightarrow \widetilde{A} / A \rightarrow 0$. To this end, we are going to establish an injective $A$-linear map $\operatorname{Hom}_{A}(\mathfrak{m}, A) \rightarrow \widetilde{A}$.

Observe first that the maps $\alpha: \Lambda=\{\lambda \in Q(A) \mid \lambda \mathfrak{m} \subset \mathfrak{m}\} \longrightarrow \operatorname{Hom}_{A}(\mathfrak{m}, \mathfrak{m})$, given by $\alpha(\lambda)(x)=\lambda x$, and $\beta: \operatorname{Hom}_{A}(\mathfrak{m}, \mathfrak{m}) \longrightarrow \operatorname{Hom}_{A}(\mathfrak{m}, A)$, induced by $\mathfrak{m} \subset A$, are both isomorphisms of $A$-modules. Indeed, both are injective and, because $Q(A)$ is a field, the surjectivity of $\alpha$ follows from $\mathfrak{m} \subset \mathfrak{m} \otimes_{A} Q(A) \cong Q(A)$. The surjectivity of $\beta$ uses that $A$ is not regular as follows. Every $A$-linear map $f: \mathfrak{m} \rightarrow A$ is injective (as $A$ is a domain) and if it does not factor via $\mathfrak{m} \subset A$, it is surjective. But if $f$ is an isomorphism, $\mathfrak{m}$ is a principal ideal, which implies that $A$ is regular.

To conclude, note that [3, Prop. 2.4] implies that $\Lambda \subset Q(A)$ is actually contained in $\widetilde{A}$. Together with the isomorphisms $\alpha$ and $\beta$ this gives the desired inclusion of $A$-modules $\operatorname{Hom}_{A}(\mathfrak{m}, A) \subset \widetilde{A}$. Using the above exact sequence, this inclusion induces an injective $A$-linear map $\operatorname{Ext}_{A}^{1}(k, A) \rightarrow \widetilde{A} / A$, hence $\mathrm{t}(A) \leq \delta(A)$.

Remark 2.2. If $A$ is regular, $\mathrm{t}(A)=1$ and $\delta(A)=0$.

Recall that the conductor ideal of $(A, \mathfrak{m}, k)$ is

$$
\mathfrak{c}=\operatorname{Ann}_{A}(\widetilde{A} / A)=\{\lambda \in A \mid \lambda \cdot \widetilde{A} \subset A\} .
$$

This is an ideal in $A$ as well as in $\widetilde{A}$, hence $\mathfrak{c} \cdot \widetilde{A}=\mathfrak{c}$ and so $\widetilde{A} / \mathfrak{c} \cong A / \mathfrak{c} \otimes_{A} \widetilde{A}$. Moreover, the map $\mathfrak{c} \longrightarrow \operatorname{Hom}_{A}(\widetilde{A}, A)$ which sends $\lambda \in \mathfrak{c}$ to multiplication by $\lambda$, is an isomorphism of $A$-modules: it is injective as $A$ is a domain and to see surjectivity we use that $\widetilde{A} \otimes_{A} Q(A) \cong Q(A)$. If $A$ is not regular, $\mathfrak{c} \neq A$ and so $\mathfrak{c} \subset \mathfrak{m}$. The multiplicity of the conductor is the length of the Artinian $A$-module $\widetilde{A} / \mathfrak{c}$, denoted

$$
c(A)=\operatorname{dim}_{k} \widetilde{A} / \mathfrak{c} .
$$

Because normalisation and completion are commuting processes for reduced excellent rings [11, Theorem 6.5], the invariants $\mathrm{t}(A), \delta(A)$ and $c(A)$ are the same for the localisation $A$ of a $\mathbb{C}$-algebra of finite type and the analytic local rings given by the same ideal. Therefore, we can use the work of Greuel [12] in our situation to obtain the following inequality.

Lemma 2.3. [12, $2.4(\mathrm{~d})]$ If the one-dimensional local Noetherian domain $A$ is the localisation of a $\mathbb{C}$-algebra of finite type, then $c(A) \leq 2 \delta(A)$.

2.2. Serre duality. Let $E$ be an integral projective curve. From the previous subsection we know that $E$ is Cohen-Macaulay. Therefore, on $E$ there exists a dualising sheaf, which we denote by $\omega_{E}$, and there exist functorial isomorphisms of 
vector spaces

$$
\operatorname{Ext}^{1}\left(\mathcal{F}, \omega_{E}\right) \cong H^{0}(\mathcal{F})^{*} \quad \text { and } \quad \operatorname{Hom}\left(\mathcal{F}, \omega_{E}\right) \cong H^{1}(\mathcal{F})^{*}
$$

for all coherent sheaves $\mathcal{F}$ on $E$, see [14, III, §7].

2.3. Relative duality. We also need relative duality for the normalisation map $\nu: \widetilde{E} \longrightarrow E$ of an integral projective curve $E$. Because $\nu$ is a finite morphism, what we need is contained in [14, III, Ex. 6.10] and worked out in detail in [4].

(a) For each quasi-coherent $\mathcal{O}_{E}$-module $\mathcal{G}$ there exists a unique (up to isomorphism) $\mathcal{O}_{\widetilde{E}}$-module $\nu ! \mathcal{G}$ such that $\nu_{*} \nu ! \mathcal{G} \cong \mathcal{H} \operatorname{om}_{E}\left(\nu_{*} \mathcal{O}_{\widetilde{E}}, \mathcal{G}\right)$. If $\mathcal{G}$ is locally free, the projection formula implies $\nu^{!} \mathcal{G} \cong \nu^{*} \mathcal{G} \otimes \nu^{!} \mathcal{O}_{E}$.

(b) For any coherent $\mathcal{O}_{\widetilde{E}}$-module $\mathcal{F}$ and any quasi-coherent $\mathcal{O}_{E}$-module $\mathcal{G}$ there exists a natural isomorphism $\nu_{*} \mathcal{H o m}_{\widetilde{E}}(\mathcal{F}, \nu ! \mathcal{G}) \stackrel{\sim}{\longrightarrow} \mathcal{H o m}_{E}\left(\nu_{*} \mathcal{F}, \mathcal{G}\right)$. Taking global sections, we obtain

$$
\operatorname{Hom}_{\widetilde{E}}(\mathcal{F}, \nu ! \mathcal{G}) \cong \operatorname{Hom}_{E}\left(\nu_{*} \mathcal{F}, \mathcal{G}\right) .
$$

2.4. Global invariants. Let $E$ be an integral projective curve over $\mathbb{C}$ and $\nu$ : $\widetilde{E} \rightarrow E$ its normalisation. By $p_{a}(E)=h^{1}\left(\mathcal{O}_{E}\right)$ and $p_{g}(E)=p_{a}(\widetilde{E})$ we denote the arithmetic and geometric genus of $E$, respectively. To define global versions of the local invariants introduced above, we look at the exact sequence

$$
0 \longrightarrow \mathcal{O}_{E} \longrightarrow \nu_{*} \mathcal{O}_{\widetilde{E}} \longrightarrow \mathcal{T} \longrightarrow 0
$$

in which $\mathcal{T}$ is a torsion sheaf with support in the singular locus of $E$. If $x \in \operatorname{sing}(E)$ and $A=\mathcal{O}_{E, x}$, this sequence localises at $x$ to $0 \longrightarrow A \longrightarrow \widetilde{A} \longrightarrow \widetilde{A} / A \longrightarrow 0$. Hence, $\operatorname{dim}_{\mathbb{C}} \mathcal{T}_{x}=\delta\left(\mathcal{O}_{E, x}\right)$ and we obtain

$$
\delta(E):=\sum_{x \in \operatorname{sing}(E)} \delta\left(\mathcal{O}_{E, x}\right)=\chi(\mathcal{T})=\chi\left(\nu_{*} \mathcal{O}_{\widetilde{E}}\right)-\chi\left(\mathcal{O}_{E}\right)=p_{a}(E)-p_{g}(E) \leq p_{a}(E) .
$$

Because $\delta(E) \geq 0$, we have $p_{g}(E) \leq p_{a}(E)$. The conductor ideal sheaf is defined as

$$
\mathcal{C}=\mathcal{A} n n_{\mathcal{O}_{E}}\left(\nu_{*} \mathcal{O}_{\widetilde{E}} / \mathcal{O}_{E}\right)
$$

The ideal sheaf $\mathcal{C} \subset \mathcal{O}_{E}$ defines a subscheme $Z \subset E$ which is supported in the singular locus $\operatorname{sing}(E)$ of $E$. Let $\widetilde{Z}=Z \times_{E} \widetilde{E}$, which is a subscheme of $\widetilde{E}$ whose ideal sheaf is denoted by $\widetilde{\mathcal{C}}$. From our local considerations, we know $\widetilde{A} / \mathfrak{c} \cong A / \mathfrak{c} \otimes_{A} \widetilde{A}$ and $\mathfrak{c} \cdot \widetilde{A}=\mathfrak{c}$. This implies that $\widetilde{\mathcal{C}}$ is the image of the canonical map $\nu^{*} \mathcal{C} \longrightarrow \mathcal{O}_{\widetilde{E}}$. This gives us a canonical map $\mathcal{C} \longrightarrow \nu_{*} \nu^{*} \mathcal{C} \longrightarrow \nu_{*} \widetilde{\mathcal{C}}$, which locally coincides with the composition $\mathfrak{c} \longrightarrow \mathfrak{c} \otimes_{A} \widetilde{A} \longrightarrow \mathfrak{c}$, which is equal to the identity. Hence, we have a canonical isomorphism $\mathcal{C} \stackrel{\sim}{\longrightarrow} \nu_{*} \widetilde{\mathcal{C}}$. Because $\mathcal{C} \cong \mathcal{H o m}_{E}\left(\nu_{*} \mathcal{O}_{\widetilde{E}}, \mathcal{O}_{E}\right)$, which we have seen locally, the definition of $\nu^{!}$implies

$$
\nu^{!} \mathcal{O}_{E} \cong \widetilde{\mathcal{C}}
$$

Lemma 2.4. $\chi\left(\nu^{!} \mathcal{O}_{E}\right) \geq 1+p_{g}(E)-2 p_{a}(E) \geq 1-2 p_{a}(E)$ 
Proof. Using the exact sequence $0 \rightarrow \widetilde{\mathcal{C}} \rightarrow \mathcal{O}_{\widetilde{E}} \rightarrow \mathcal{O}_{\widetilde{Z}} \rightarrow 0$, we obtain

$$
\chi\left(\nu ! \mathcal{O}_{E}\right)=\chi(\widetilde{\mathcal{C}})=\chi\left(\mathcal{O}_{\widetilde{E}}\right)-\chi\left(\mathcal{O}_{\widetilde{Z}}\right)=1-p_{g}(E)-\chi\left(\mathcal{O}_{\widetilde{Z}}\right) .
$$

Because $\chi\left(\mathcal{O}_{\widetilde{Z}}\right)=\sum_{x \in \operatorname{sing}(E)} c\left(\mathcal{O}_{E, x}\right)$, Lemma 2.3 implies

$$
\chi\left(\mathcal{O}_{\widetilde{Z}}\right) \leq 2 \sum_{x \in \operatorname{sing}(E)} \delta\left(\mathcal{O}_{E, x}\right)=2 \delta(E)=2 p_{a}(E)-2 p_{g}(E),
$$

hence $\chi\left(\nu^{!} \mathcal{O}_{E}\right) \geq 1+p_{g}(E)-2 p_{a}(E) \geq 1-2 p_{a}(E)$.

2.5. Families of simple sheaves. The families of sheaves considered in the definition of the functor $\underline{\mathrm{M}}_{X / S}^{(n, d)}$ are simple on each fibre. We also need that for such families the canonical morphism $\mathcal{O}_{S} \rightarrow p_{*} \mathcal{H} \operatorname{om}_{X}(\mathcal{F}, \mathcal{F})$ is an isomorphism. This can be found in [15, Lemma 4.6.3] and in [1, Cor. 5.3] for the case of schemes, which inspired the proof we present for the analytic case.

Lemma 2.5. If $p: X \longrightarrow S$ be a proper and flat morphism of complex spaces and $\mathcal{F}$ a coherent sheaf on $X$, flat over $S$, such that $\mathcal{F}_{s}$ is simple for all $s \in S$, then

$$
\mathcal{O}_{S} \stackrel{\sim}{\longrightarrow} p_{*} \mathcal{H o m}_{X}(\mathcal{F}, \mathcal{F}) \text {. }
$$

Proof. The canonical morphism $\mathcal{O}_{S} \rightarrow p_{*} \mathcal{H} \operatorname{mom}_{X}(\mathcal{F}, \mathcal{F})$ is injective because $\mathcal{F}$ is $S$ flat. Restricting the exact sequence $0 \rightarrow \mathcal{O}_{S} \rightarrow p_{*} \mathcal{H o m}_{X}(\mathcal{F}, \mathcal{F}) \rightarrow \mathcal{T} \rightarrow 0$ to a point $s \in S$, we obtain the exact sequence

$$
\mathbb{C} \stackrel{\alpha}{\longrightarrow} p_{*} \mathcal{H o m}_{X}(\mathcal{F}, \mathcal{F})(s) \longrightarrow \mathcal{T}(s) \longrightarrow 0 \text {. }
$$

The base-change morphism $\varphi_{s}: p_{*} \mathcal{H} \operatorname{om}_{X}(\mathcal{F}, \mathcal{F})(s) \rightarrow \operatorname{Hom}_{X_{s}}\left(\mathcal{F}_{s}, \mathcal{F}_{s}\right)$ composed with $\alpha$ yields the canonical map $\mathbb{C} \rightarrow \operatorname{Hom}_{X_{s}}\left(\mathcal{F}_{s}, \mathcal{F}_{s}\right)$, which was supposed to be an isomorphism. By [6, Satz 2 (ii)] the surjectivity of $\varphi_{s}$ already implies that it is an isomorphism. Hence, $\alpha$ is an isomorphism as well and so $\mathcal{T}(s)=0$ for all $s \in S$. As all local rings $\mathcal{O}_{S, s}$ are Noetherian, this is sufficient to conclude that $\mathcal{T}=0$, which implies the claim.

\section{A VANishing THEOREM FOR SIMPLE SHEAVES}

Serre's Vanishing Theorem says that twists of a coherent sheaf with a sufficiently high tensor power of an ample line bundle have vanishing higher cohomology. How large this tensor power needs to be, in general depends on the underlying projective variety and the coherent sheaf. We will prove here a version for simple sheaves on complex projective curves in which the vanishing is guaranteed above a threshold which only depends on the arithmetic genus of the curve as well as the rank and the Euler characteristic of the coherent sheaf.

Throughout this section, we fix integers $g \geq 0, n \geq 0$ and $d$. All curves will be curves over the field $\mathbb{C}$. The main result of this section is the following theorem. 
Theorem 3.1. There exists an integer $m_{0}=m_{0}(g, n, d)$, depending only on $g, n, d$, such that for all integral projective curves $E$ of arithmetic genus $g$, for all simple coherent sheaves $\mathcal{F}$ on $E$ with $\operatorname{rk}(\mathcal{F})=n$ and $\chi(\mathcal{F})=d$, for all line bundles $\mathcal{L}$ of degree 1 on $E$ and for all $m \geq m_{0}$ we have

$$
H^{1}\left(\mathcal{F} \otimes \mathcal{L}^{\otimes m}\right)=0 .
$$

As a tool in our proofs we use a stability structure on the abelian category of coherent sheaves on an integral projective curve $E$. It is convenient to use the framework of [19], where stability is defined with the aid of a linearly independent system of additive functions. We exclusively work with the pair of additive functions $($ rk, $\chi$ ). We define $\mu(\mathcal{F})=\chi(\mathcal{F}) / \operatorname{rk}(\mathcal{F})$ when $\operatorname{rk}(\mathcal{F})>0$ and let $\mu(\mathcal{F})=\infty$ if $\operatorname{rk}(\mathcal{F})=0$. A non-zero coherent sheaf $\mathcal{F}$ is called semi-stable if $\mu(\mathcal{G}) \leq \mu(\mathcal{F})$ for all non-zero subsheaves $\mathcal{G}$. In particular, all torsion sheaves are semi-stable and semi-stable sheaves of positive rank are automatically torsion free. Moreover, it is not hard to see that all torsion free sheaves of rank one are semi-stable. The key facts we need are

(i) If $\mathcal{F}, \mathcal{G}$ are semi-stable and $\mu(\mathcal{F})>\mu(\mathcal{G})$ then $\operatorname{Hom}(\mathcal{F}, \mathcal{G})=0$.

(ii) For every non-zero coherent sheaf $\mathcal{F}$ there exists a filtration, known as the Harder-Narasimhan filtration (HNF), $0=\mathcal{F}_{0} \subset \mathcal{F}_{1} \subset \ldots \subset \mathcal{F}_{k-1} \subset \mathcal{F}_{k}=\mathcal{F}$, such that the factors $\mathcal{A}_{i}=\mathcal{F}_{i} / \mathcal{F}_{i-1}$ are semi-stable and

$$
\mu_{\max }(\mathcal{F})=\mu\left(\mathcal{A}_{1}\right)>\mu\left(\mathcal{A}_{2}\right)>\ldots>\mu\left(\mathcal{A}_{k-1}\right)>\mu\left(\mathcal{A}_{k}\right)=\mu_{\min }(\mathcal{F}) .
$$

This filtration is unique and so $\mu_{\max }$ and $\mu_{\min }$ are well-defined. We easily obtain $\mu_{\max }(\mathcal{F}) \geq \mu(\mathcal{F}) \geq \mu_{\text {min }}(\mathcal{F})$.

Proofs are pretty well-known by now and can be found in [19]. Note that the abelian category of coherent sheaves on $E$ is Noetherian (i.e. ascending chains of subobjects eventually stabilise) and, with respect to the stability defined above, it is weakly Artinian in the sense of [19], i.e. there is no infinite descending chain of coherent subsheaves with strictly increasing slopes.

Remark 3.2. The vanishing in Theorem 3.1 is easily obtained for semi-stable sheaves $\mathcal{F}$. If $\mathcal{F}$ is torsion, the vanishing is trivial. If $\mathcal{F}$ is torsion free, we set $m_{0}=g-1-\frac{d}{n}$. Then we have $\mu(\mathcal{F})=\frac{d}{n}>2 g-2-m+1-g=\mu\left(\omega_{E} \otimes \mathcal{L}^{\otimes(-m)}\right)$, whenever $m>m_{0}$. Because $\omega_{E}$ is torsion free of rank one, for $\mathcal{F}$ semi-stable we obtain $\operatorname{Hom}\left(\mathcal{F}, \omega_{E} \otimes \mathcal{L}^{\otimes(-m)}\right)=0$. Using Serre-duality this implies

$$
H^{1}\left(\mathcal{F} \otimes \mathcal{L}^{\otimes m}\right)=\operatorname{Hom}\left(\mathcal{F} \otimes \mathcal{L}^{\otimes m}, \omega_{E}\right)^{*}=\operatorname{Hom}\left(\mathcal{F}, \omega_{E} \otimes \mathcal{L}^{\otimes(-m)}\right)^{*}=0 .
$$

If $\mathcal{F}$ is not semi-stable, we need to guarantee such a vanishing for all semi-stable quotients that can appear in the Harder-Narasimhan filtration of $\mathcal{F}$, but their slopes are no longer equal to $\frac{d}{n}$. Our main task will be to find a lower bound for these slopes. 
Lemma 3.3. Let $E$ be an integral projective curve, $\mathcal{F} \in \operatorname{Coh}(E)$ a torsion free sheaf and $\mathcal{L} \in \operatorname{Pic}(E)$, then

$$
\mu(\mathcal{F} \otimes \mathcal{L})=\mu(\mathcal{F})+\operatorname{deg}(\mathcal{L})
$$

Proof. Because $\operatorname{rk}(\mathcal{F})>0$, the statement is equivalent to the equation

$$
\chi(\mathcal{F} \otimes \mathcal{L})=\chi(\mathcal{F})+\operatorname{deg}(\mathcal{L}) \operatorname{rk}(\mathcal{F}) .
$$

This equation holds true for arbitrary coherent sheaves on $E$. It is obvious if $\mathcal{F}$ is a torsion sheaf. Because $\chi$ and rk are additive functions, both sides of equation (1) are additive. Looking at the exact sequence $0 \rightarrow \operatorname{tors}(\mathcal{F}) \rightarrow \mathcal{F} \rightarrow \mathcal{G} \rightarrow 0$, where $\mathcal{G}$ is a torsion free sheaf and $\operatorname{tors}(\mathcal{F})$ is the torsion subsheaf of $\mathcal{F}$, we see that it is sufficient to prove equality (1) for torsion free sheaves.

If $\mathcal{F}$ is torsion free and $\nu: \widetilde{E} \rightarrow E$ is the normalisation, we let $\widetilde{\mathcal{F}}=\nu^{*}(\mathcal{F}) /$ torsion and obtain an exact sequence $0 \rightarrow \mathcal{F} \rightarrow \nu_{*} \widetilde{\mathcal{F}} \rightarrow \mathcal{T} \rightarrow 0$, where $\mathcal{T}$ is a torsion sheaf on $E$. Hence it is enough to show equation (1) for sheaves $\mathcal{F}=\nu_{*} \widetilde{\mathcal{F}}$, where $\widetilde{\mathcal{F}}$ is a vector bundle on the smooth curve $\widetilde{E}$. The projection formula gives an isomorphism $\nu_{*} \widetilde{\mathcal{F}} \otimes \mathcal{L} \cong \nu_{*}\left(\widetilde{\mathcal{F}} \otimes \nu^{*} \mathcal{L}\right)$. The usual Riemann-Roch for vector bundles on $\widetilde{E}$ implies

$$
\begin{aligned}
\chi\left(\nu_{*} \widetilde{\mathcal{F}} \otimes \mathcal{L}\right) & =\chi\left(\nu_{*}\left(\widetilde{\mathcal{F}} \otimes \nu^{*} \mathcal{L}\right)\right)=\chi\left(\widetilde{\mathcal{F}} \otimes \nu^{*} \mathcal{L}\right)=\chi(\widetilde{\mathcal{F}})+\operatorname{deg}\left(\nu^{*} \mathcal{L}\right) \operatorname{rk}(\widetilde{\mathcal{F}}) \\
& =\chi\left(\nu_{*} \widetilde{\mathcal{F}}\right)+\operatorname{deg}(\mathcal{L}) \operatorname{rk}\left(\nu_{*} \widetilde{\mathcal{F}}\right)
\end{aligned}
$$

from which the claim follows.

Remark 3.4. Because tensor product with a line bundle $\mathcal{L}$ is an exact functor, the HN factors of $\mathcal{F} \otimes \mathcal{L}$ are $\mathcal{A}_{i} \otimes \mathcal{L}$, when $\mathcal{A}_{i}$ are the HN factors of $\mathcal{F}$. As a consequence, the equation in Lemma 3.3 holds for $\mu_{\max }$ and $\mu_{\min }$ as well.

Lemma 3.5. Let $E$ be an integral projective curve, $\mathcal{A} \in \operatorname{Coh}(E)$ torsion free, $q \in \mathbb{Z}$ and $\mathcal{L} \in \operatorname{Pic}(E)$ such that $\mu(\mathcal{A})<-q \operatorname{deg}(\mathcal{L})$, then $\operatorname{Hom}\left(\mathcal{A}, \omega_{E} \otimes \mathcal{L}^{-q}\right) \neq 0$.

Proof. By Lemma 3.3 we have $\mu\left(\mathcal{A} \otimes \mathcal{L}^{q}\right)=\mu(\mathcal{A})+q \operatorname{deg}(\mathcal{L})<0$, hence $\chi\left(\mathcal{A} \otimes \mathcal{L}^{q}\right)<0$ and this implies $0 \neq H^{1}\left(\mathcal{A} \otimes \mathcal{L}^{q}\right)=\operatorname{Hom}\left(\mathcal{A} \otimes \mathcal{L}^{q}, \omega_{E}\right)^{*}=\operatorname{Hom}\left(\mathcal{A}, \omega_{E} \otimes \mathcal{L}^{-q}\right)^{*}$, using Serre-duality.

Lemma 3.6. Let $g \geq 0$ be an integer. Then, for all integral projective curves $E$ of arithmetic genus $g$, for all line bundles $\mathcal{L}$ of degree one on $E$ and for all $q \geq g^{2}+4 g-2$

$$
\operatorname{Hom}\left(\omega_{E}, \mathcal{L}^{q}\right) \neq 0 .
$$

Proof. Let $\nu: \widetilde{E} \rightarrow E$ be the normalisation. As $\nu^{!} \omega_{E} \cong \omega_{\widetilde{E}}$ (see [14, III Ex. 7.2]) and $\left(\nu_{*}, \nu^{!}\right)$is an adjoint pair, there is a natural morphism of $\mathcal{O}_{E^{-}}$modules $\nu_{*} \omega_{\widetilde{E}} \rightarrow \omega_{E}$. Because $\omega_{\widetilde{E}}$ is torsion free and the natural morphism is an isomorphism on the regular locus of $E$, we obtain an exact sequence

$$
0 \longrightarrow \nu_{*} \omega_{\widetilde{E}} \longrightarrow \omega_{E} \longrightarrow \mathcal{T} \longrightarrow 0
$$


in which $\mathcal{T}$ is a torsion sheaf with support in the singular locus of $E$. Applying the functor $\operatorname{Hom}\left(\cdot, \mathcal{L}^{q}\right)$ produces the exact sequence

$$
0 \longrightarrow \operatorname{Hom}\left(\omega_{E}, \mathcal{L}^{q}\right) \longrightarrow \operatorname{Hom}\left(\nu_{*} \omega_{\widetilde{E}}, \mathcal{L}^{q}\right) \longrightarrow \operatorname{Ext}^{1}\left(\mathcal{T}, \mathcal{L}^{q}\right)
$$

We first give an upper bound, not depending on $q$, for the dimension of the third term in this sequence. Then we will show that the dimension of the second term grows unboundedly with $q$.

For each singular point $x \in E$ we denote by $\ell_{x}$ the dimension of the stalk $\mathcal{T}_{x}$, so that $\chi(\mathcal{T})=\sum_{x \in \operatorname{sing}(E)} \ell_{x}$. Using a Jordan-Hölder filtration for the $\mathcal{O}_{E, x}$-module $\mathcal{T}_{x}$, we obtain $\operatorname{dim} \mathcal{E} \times \mathrm{xt}^{1}\left(\mathcal{T}, \mathcal{L}^{q}\right)_{x}=\operatorname{dim} \operatorname{Ext}_{\mathcal{O}_{E, x}}^{1}\left(\mathcal{T}_{x}, \mathcal{O}_{E, x}\right) \leq \ell_{x} \cdot \mathrm{t}\left(\mathcal{O}_{E, x}\right)$. Because $\operatorname{Ext}^{1}\left(\mathcal{T}, \mathcal{L}^{q}\right)=H^{0}\left(\mathcal{E} x^{1}\left(\mathcal{T}, \mathcal{L}^{q}\right)\right)$, Lemma 2.1 implies now

$$
\operatorname{dim} \operatorname{Ext}^{1}\left(\mathcal{T}, \mathcal{L}^{q}\right) \leq \sum_{x \in \operatorname{sing}(E)} \ell_{x} \delta\left(\mathcal{O}_{E, x}\right) \leq \delta(E) \sum_{x \in \operatorname{sing}(E)} \ell_{x}=\delta(E) \chi(\mathcal{T})
$$

From (2) we get $\chi(\mathcal{T})=\chi\left(\omega_{E}\right)-\chi\left(\nu_{*} \omega_{\widetilde{E}}\right)=p_{a}(E)-p_{g}(E) \leq p_{a}(E)=g$. We have seen in Subsection 2.4 that $\delta(E) \leq p_{a}(E)=g$ and therefore we can conclude

$$
\operatorname{dim} \operatorname{Ext}^{1}\left(\mathcal{T}, \mathcal{L}^{q}\right) \leq g^{2}
$$

To study the second term in the sequence (3) we first use relative duality to obtain

$$
\begin{aligned}
\operatorname{Hom}_{E}\left(\nu_{*} \omega_{\widetilde{E}}, \mathcal{L}^{q}\right) & =\operatorname{Hom}_{\widetilde{E}}\left(\omega_{\widetilde{E}}, \nu^{!} \mathcal{L}^{q}\right)=\operatorname{Hom}_{\widetilde{E}}\left(\omega_{\widetilde{E}}, \nu^{*} \mathcal{L}^{q} \otimes \nu^{!} \mathcal{O}\right) \\
& =H^{0}\left(\nu^{*} \mathcal{L}^{q} \otimes \omega_{\widetilde{E}}^{\vee} \otimes \nu^{!} \mathcal{O}\right) .
\end{aligned}
$$

Because $\nu^{*} \mathcal{L}^{q} \otimes \omega_{\widetilde{E}}^{\vee}$ is an invertible sheaf and $\nu^{!} \mathcal{O}$ has rank one, with the aid of Lemma 3.3 and Lemma 2.4, and using $g=p_{a}(E) \geq p_{g}(E)$, we obtain

$$
\begin{aligned}
h^{0}\left(\nu^{*} \mathcal{L}^{q} \otimes \omega_{\widetilde{E}}^{\vee} \otimes \nu^{!} \mathcal{O}\right) & \geq \chi\left(\nu^{*} \mathcal{L}^{q} \otimes \omega_{\widetilde{E}}^{\vee} \otimes \nu^{!} \mathcal{O}\right)=\chi\left(\nu^{!} \mathcal{O}\right)+\operatorname{deg}\left(\nu^{*} \mathcal{L}^{q} \otimes \omega_{\widetilde{E}}^{\vee}\right) \\
& =\chi\left(\nu^{!} \mathcal{O}\right)+q-2 p_{g}(E)+2 \geq 1-2 p_{a}(E)+q-2 p_{g}(E)+2 \\
& \geq q+3-4 g \geq g^{2}+1
\end{aligned}
$$

whenever $q \geq g^{2}+4 g-2$. The sequence (3) implies now that $\operatorname{Hom}\left(\omega_{E}, \mathcal{L}^{q}\right) \neq 0$ for all $q \geq g^{2}+4 g-2$.

Proposition 3.7. Let $E$ be an integral projective curve of arithmetic genus $g$ and let $\mathcal{F} \in \operatorname{Coh}(E)$ be a simple torsion free sheaf, then

$$
\mu(\mathcal{F})-\mu_{\min }(\mathcal{F}) \leq g^{2}+4 g .
$$

Proof. First observe that Lemma 3.3 implies that $\mu(\mathcal{F})-\mu_{\min }(\mathcal{F})$ remains unchanged when $\mathcal{F}$ is twisted by a line bundle. After twisting with a line bundle we can always achieve that $0<\mu_{\max }(\mathcal{F}) \leq 1$. Hence, we can make this assumption without loss of generality. Let $0=\mathcal{F}_{0} \subset \mathcal{F}_{1} \subset \ldots \subset \mathcal{F}_{k-1} \subset \mathcal{F}_{k}=\mathcal{F}$ be the HNF of $\mathcal{F}$ with semi-stable factors $\mathcal{A}_{i}=\mathcal{F}_{i} / \mathcal{F}_{i-1}$. We have $\mu_{\max }(\mathcal{F})=\mu\left(\mathcal{A}_{1}\right)$ and $\mu_{\min }(\mathcal{F})=\mu\left(\mathcal{A}_{k}\right)$.

For a proof by contradiction we assume $\mu\left(\mathcal{A}_{k}\right)<1-4 g-g^{2}$. Let $\mathcal{L}$ be a line bundle of degree one on $E$. By Lemma 3.5 with $q=g^{2}+4 g-1$ there exists a non-vanishing 
morphism of sheaves $\alpha: \mathcal{A}_{k} \rightarrow \omega_{E} \otimes \mathcal{L}^{1-4 g-g^{2}}$. From Lemma 3.6 with $q=g^{2}+4 g-1>$ $g^{2}+4 g-2$ we obtain a non-zero morphism $\beta: \omega_{E} \otimes \mathcal{L}^{1-4 g-g^{2}} \rightarrow \mathcal{O}$. Finally, because we assumed $\mu\left(\mathcal{A}_{1}\right)=\mu_{\max }(\mathcal{F})>0$, we have $h^{0}\left(\mathcal{A}_{1}\right) \geq \chi\left(\mathcal{A}_{1}\right)>0$ and so there exists a non-zero morphism $\gamma: \mathcal{O} \rightarrow \mathcal{A}_{1}$. Together with the epimorphism $\mathcal{F} \rightarrow \mathcal{A}_{k}$ and the inclusion $\mathcal{A}_{1} \subset \mathcal{F}$, we obtain a chain of non-zero morphisms

$$
\mathcal{F} \rightarrow \mathcal{A}_{k} \stackrel{\alpha}{\longrightarrow} \omega_{E} \otimes \mathcal{L}^{1-4 g-g^{2}} \stackrel{\beta}{\longrightarrow} \mathcal{O} \stackrel{\gamma}{\longrightarrow} \mathcal{A}_{1} \subset \mathcal{F} .
$$

Because any morphism from a torsion free rank one sheaf to a torsion free sheaf is automatically a monomorphism, $\beta$ and $\gamma$ are monomorphism and so the composition (5) is a non-zero endomorphism of $\mathcal{F}$. As $\mathcal{F}$ was assumed to be simple, this composition must be an isomorphism and so also each morphism in the chain (5). In particular, $\mathcal{F} \cong \mathcal{O}$ and so $\mu_{\min }(\mathcal{F})=\mu(\mathcal{F})=\chi(\mathcal{O})=1-g \geq 1-4 g-g^{2}$. This contradicts our assumption $\mu\left(\mathcal{A}_{k}\right)<1-4 g-g^{2}$, thus proves that we have $\mu_{\min }(\mathcal{F})=$ $\mu\left(\mathcal{A}_{k}\right) \geq 1-4 g-g^{2}$. Using $\mu(\mathcal{F}) \leq \mu_{\max }(\mathcal{F}) \leq 1$, we obtain $\mu(\mathcal{F})-\mu_{\min }(\mathcal{F}) \leq g^{2}+4 g$, as required.

Proof of Theorem 3.1. Let $E, \mathcal{F}$ and $\mathcal{L}$ be as in the formulation of the theorem. If the simple sheaf $\mathcal{F}$ is not torsion free, the torsion subsheaf of $\mathcal{F}$ is non-zero and so contains a rank one sky-scraper $\mathbb{C}_{s}$ supported at some point $s \in E$. On the other hand, by restricting to $s$ we always get an epimorphism $\mathcal{F} \rightarrow \mathbb{C}_{s}$. The composition $\mathcal{F} \rightarrow \mathbb{C}_{s} \subset \mathcal{F}$ is a non-trivial endomorphism, hence an isomorphism. In particular, $\mathcal{F} \cong \mathbb{C}_{s}$ is a torsion sheaf. In this case, the claimed vanishing is trivial for all $m \in \mathbb{Z}$, because the support of $\mathcal{F} \otimes \mathcal{L}^{\otimes m}$ is of dimension zero.

For the rest of the proof we assume that $\mathcal{F}$ is a simple torsion free sheaf. Let $0=\mathcal{F}_{0} \subset \mathcal{F}_{1} \subset \ldots \subset \mathcal{F}_{k-1} \subset \mathcal{F}_{k}=\mathcal{F}$ be the HNF of $\mathcal{F}$ with semi-stable factors $\mathcal{A}_{i}=\mathcal{F}_{i} / \mathcal{F}_{i-1}$. Recall that $\mu(\mathcal{F})=\frac{d}{n}$. Let $m_{0}=\left\lfloor g^{2}+5 g-\frac{d}{n}\right\rfloor$ and $m \geq m_{0}$. Because $\mu\left(\omega_{E} \otimes \mathcal{L}^{\otimes(-m)}\right)=g-1-m \leq g-1-m_{0}<g-1-\left(g^{2}+5 g-1-\mu(\mathcal{F})\right)=$ $\mu(\mathcal{F})-\left(g^{2}+4 g\right) \leq \mu_{\min }(\mathcal{F})$ by Proposition 3.7, the semi-stability of the factors $\mathcal{A}_{i}$ implies $\operatorname{Hom}\left(\mathcal{A}_{i}, \omega_{E} \otimes \mathcal{L}^{\otimes(-m)}\right)=0$. From the exact sequences $0 \rightarrow \mathcal{F}_{i-1} \rightarrow$ $\mathcal{F}_{i} \rightarrow \mathcal{A}_{i} \rightarrow 0$ we obtain then $\operatorname{Hom}\left(\mathcal{F}, \omega_{E} \otimes \mathcal{L}^{\otimes(-m)}\right)=0$. Serre-duality implies now $H^{1}\left(\mathcal{F} \otimes \mathcal{L}^{\otimes m}\right)=\operatorname{Hom}\left(\mathcal{F} \otimes \mathcal{L}^{\otimes m}, \omega_{E}\right)^{*}=\operatorname{Hom}\left(\mathcal{F}, \omega_{E} \otimes \mathcal{L}^{\otimes(-m)}\right)^{*}=0$, as required.

Remark 3.8. A moduli problem for coherent sheaves in the category of schemes will be bounded if the Castelnuovo-Mumford regularity of these sheaves is bounded. The corresponding moduli space is then of finite type or even quasi-projective. The significance of Theorem 3.1 in the present situation is highlighted in Remark 4.5.

\section{Proof of the MAIN THEOREM}

The family $p: X \rightarrow S$ is assumed to satisfy the conditions formulated in the Introduction. In addition to the notation introduced there we denote by $\Sigma=i(S)$ the image of the section $i$, which is a divisor on $X$. For any object $T \rightarrow S$ in $\operatorname{An}_{S}$, 
we continue to denote the projection by $q: X_{T} \rightarrow T$ and denote by $\Sigma_{T} \subset X_{T}$ the induced section. For any coherent sheaf $\mathcal{F}$ on $X_{T}$ we write $\mathcal{F}(m):=\mathcal{F} \otimes \mathcal{O}\left(m \Sigma_{T}\right)$.

Theorem 4.1. If $n$ and $d$ are coprime, then the functor $\underline{M}_{X / S}^{(n, d)}$ is representable.

In order to prove the existence of a fine moduli space $\mathrm{M}_{X / S}^{(n, d)}$ we apply a result of Kosarew and Okonek [16]. If we can show that the functor $\underline{\mathrm{M}}_{X / S}^{(n, d)}$ is a sheaf in the analytic topology, it follows from their Theorem 6.4 and Remark 6.7 that this functor is representable.

The separatedness of the functor $\underline{\mathrm{M}}_{X / S}^{(n, d)}$, which is part of the sheaf property, means that for any morphism $T \rightarrow S$, two families $\mathcal{F}, \mathcal{G} \in \underline{\mathrm{M}}_{X / S}^{(n, d)}(T)$ coincide if they do so locally. More precisely, this means that if for an open cover $\left\{T_{i}\right\}$ of $T$ we have $\left.\left.\mathcal{F}\right|_{X_{T_{i}}} \sim \mathcal{G}\right|_{X_{T_{i}}}$, then $\mathcal{F} \sim \mathcal{G}$. The following lemma implies that the functor $\underline{\mathrm{M}}_{X / S}^{(n, d)}$ is separated. The equivalence relation $\sim$ corresponds precisely to the construction of a separated functor, see [16, Remark (6.7)], or [21, Section 2.3].

Lemma 4.2. Let $p: X \longrightarrow S$ be a proper and flat morphism of complex spaces, $\mathcal{F}$ and $\mathcal{G}$ two $S$-flat coherent sheaves on $X$ such that for all points $s \in S$

(i) $\mathcal{F}_{s}$ is simple;

(ii) there exists an open neighbourhood $U$ of $s \in S$ such that $\mathcal{F}_{U} \cong \mathcal{G}_{U}$; then there exists a line bundle $\mathcal{L} \in \operatorname{Pic}(S)$ such that $\mathcal{G} \cong \mathcal{F} \otimes p^{*} \mathcal{L}$.

Proof. Assumption (ii), in which $\mathcal{F}_{U}, \mathcal{G}_{U}$ denote restrictions to the open subset $X_{U} \subset X$, implies that for any $s \in S$ there exists an isomorphism $\varphi: \mathcal{F}_{s} \rightarrow \mathcal{G}_{s}$ which generates the one-dimensional vector space $\operatorname{Hom}_{X_{s}}\left(\mathcal{F}_{s}, \mathcal{G}_{s}\right)$. We obtain a commutative diagram

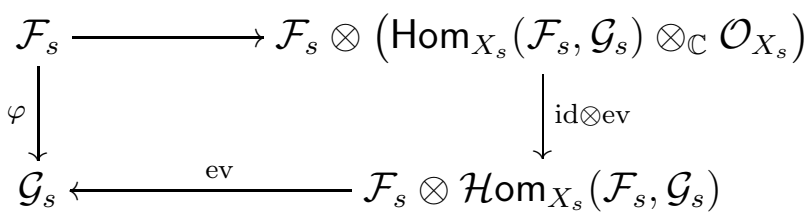

in which the upper horizontal arrow is the isomorphism induced by $\varphi$. This implies that the canonical morphism

$$
\mathcal{F}_{s} \otimes\left(\operatorname{Hom}_{X_{s}}\left(\mathcal{F}_{s}, \mathcal{G}_{s}\right) \otimes_{\mathbb{C}} \mathcal{O}_{X_{s}}\right) \longrightarrow \mathcal{G}_{s}
$$

is an isomorphism for all $s \in S$.

Because $\left.p_{*} \mathcal{H o m}_{X}(\mathcal{F}, \mathcal{G})\right|_{U} \cong p_{U *} \mathcal{H o m}_{X_{U}}\left(\mathcal{F}_{U}, \mathcal{G}_{U}\right)$, where $p_{U}: X_{U} \rightarrow U$ is the restriction of $p$ to $U \subset S$, assumptions (i) and (ii) and Lemma 2.5 imply that $\left.p_{*} \mathcal{H o m}_{X}(\mathcal{F}, \mathcal{G})\right|_{U} \cong \mathcal{O}_{U}$. This means that $\mathcal{L}:=p_{*} \mathcal{H o m}_{X}(\mathcal{F}, \mathcal{G})$ is a line bundle on $S$. Thus, the following composition of canonical morphisms of sheaves on $X$

$$
\mathcal{F} \otimes p^{*} p_{*} \mathcal{H o m}_{X}(\mathcal{F}, \mathcal{G}) \longrightarrow \mathcal{F} \otimes \mathcal{H} \operatorname{om}_{X}(\mathcal{F}, \mathcal{G}) \longrightarrow \mathcal{G}
$$


is an isomorphism on all fibres $X_{s}$. Because $\mathcal{G}$ is $S$-flat, this is sufficient to conclude that $\mathcal{F} \otimes p^{*} \mathcal{L} \longrightarrow \mathcal{G}$ is an isomorphism.

We shall see below that the sheaf property will follow if we can show that there exists a certain twisted line bundle on $T$. For its construction we denote

$$
\widetilde{\mathrm{M}}_{X / S}^{(n, d)}(T)=\left\{\mathcal{F} \in \operatorname{Coh}\left(X_{T}\right) \mid \begin{array}{l}
\mathcal{F} \text { is } T \text {-flat, } \mathcal{F}_{t} \text { is simple } \\
\operatorname{rk}\left(\mathcal{F}_{t}\right)=n, \chi\left(\mathcal{F}_{t}\right)=d \quad \forall t \in T
\end{array}\right\} \subset \operatorname{Coh}\left(X_{T}\right),
$$

so that $\underline{\mathrm{M}}_{X / S}^{(n, d)}(T)=\widetilde{\mathrm{M}}_{X / S}^{(n, d)}(T) / \sim$. We use functors $\mathbb{L}_{T}: \widetilde{\mathrm{M}}_{X / S}^{(n, d)}(T) \rightarrow \operatorname{Coh}(T)$ which are defined as follows. Fix an integer $m \geq m_{0}$ (see Theorem 3.1) and let $a, b$ be integers such that $a(d+m n)+b(d+(m+1) n)=1$. Such integers exist if $\operatorname{gcd}(n, d)=1$. For any $T \in \operatorname{An}_{S}$ and $\mathcal{F} \in \widetilde{\mathrm{M}}_{X / S}^{(n, d)}(T)$ we define

$$
\mathbb{L}_{T}(\mathcal{F})=\left(\operatorname{det}\left(q_{*} \mathcal{F}(m)\right)\right)^{\otimes a} \otimes\left(\operatorname{det}\left(q_{*} \mathcal{F}(m+1)\right)\right)^{\otimes b} .
$$

Lemma 4.3. The functors $\mathbb{L}_{T}: \widetilde{\mathrm{M}}_{X / S}^{(n, d)}(T) \rightarrow \operatorname{Coh}(T)$ have the following properties.

(a) If $\mathcal{F} \in \widetilde{\mathrm{M}}_{X / S}^{(n, d)}(T)$, then $\mathbb{L}_{T}(\mathcal{F}) \in \operatorname{Pic}(T)$.

(b) For $\lambda \in H^{0}\left(\mathcal{O}_{T}\right)$ and $\mathcal{F} \in \widetilde{\mathrm{M}}_{X / S}^{(n, d)}(T)$ we have $\mathbb{L}_{T}\left(q^{*}(\lambda) \cdot \operatorname{id}_{\mathcal{F}}\right)=\lambda \cdot \operatorname{id}_{\mathbb{L}_{T}(\mathcal{F})}$.

(c) If $u: T^{\prime} \subset T$ is an open subset and $v: X_{T^{\prime}} \subset X_{T}$, then $u^{*} \circ \mathbb{L}_{T} \cong \mathbb{L}_{T^{\prime}} \circ v^{*}$.

Proof. (a) By Theorem 3.1 we have $H^{1}\left(\mathcal{F}(m)_{t}\right)=H^{1}\left(\mathcal{F}(m+1)_{t}\right)=0$ for all $t \in T$. From [5, III.3.9] we obtain that $q_{*} \mathcal{F}(m)$ and $q_{*} \mathcal{F}(m+1)$ are locally free, and the result follows. Moreover, it follows that the ranks of these locally free sheaves are equal to $\chi\left(\mathcal{F}(m)_{t}\right)=d+m n$ and $\chi\left(\mathcal{F}(m+1)_{t}\right)=d+(m+1) n$, respectively. This will be used in the next part of the proof.

(b) The functor $q_{*}$ sends $q^{*}(\lambda) \cdot \operatorname{id}_{\mathcal{F}(m)}$ to $\lambda \cdot \operatorname{id}_{q_{*} \mathcal{F}(m)}$ which is sent to $\lambda^{d+m n} \cdot$ id by the functor det. Therefore, $\mathbb{L}_{T}\left(q^{*}(\lambda) \cdot \mathrm{id}_{\mathcal{F}}\right)=\lambda^{a(d+m n)+b(d+(m+1) n)} \cdot \operatorname{id}_{\mathbb{L}_{T}(\mathcal{F})}=\lambda \cdot \operatorname{id}_{\mathbb{L}_{T}(\mathcal{F})}$.

(c) This is clear because tensor product and taking the determinant is compatible with pull-back and, if $q^{\prime}$ denotes the restriction of $q$ to $X_{T^{\prime}}, u^{*} \circ q_{*}=q_{*}^{\prime} \circ v^{*}$ holds.

Proposition 4.4. The functor $\underline{\mathrm{M}}_{X / S}^{(n, d)}$ is a sheaf in the analytic topology.

Proof. In order to prove that the separated functor $\underline{\mathrm{M}}_{X / S}^{(n, d)}$ is a sheaf it is sufficient to prove the following. For each $T \in \mathrm{An}_{S}$, for each open cover $\left\{T_{i}\right\}$ of $T$ and any $\mathcal{F}_{i} \in \underline{\mathrm{M}}_{X / S}^{(n, d)}\left(T_{i}\right)$ which satisfy $\left.\left.\mathcal{F}_{j}\right|_{X_{i j}} \cong \mathcal{F}_{i}\right|_{X_{i j}}$ for all $i, j$, there exists $\mathcal{F} \in \underline{\mathrm{M}}_{X / S}^{(n, d)}(T)$ such that $\left.\mathcal{F}\right|_{X_{i}} \sim \mathcal{F}_{i}$. Here we abbreviate $X_{i}:=X_{T_{i}}$ and $X_{i j}:=\left(T_{i} \cap T_{j}\right) \times_{S} X$. More explicitly, the equivalence $\left.\mathcal{F}\right|_{X_{i}} \sim \mathcal{F}_{i}$ means that there exist line bundles $\mathcal{L}_{i}$ on $T_{i}$ such that $\left.\mathcal{F}\right|_{X_{i}} \cong \mathcal{F}_{i} \otimes q^{*} \mathcal{L}_{i}$.

To prove this, we denote by $\varphi_{i j}:\left.\left.\mathcal{F}_{j}\right|_{X_{i j}} \rightarrow \mathcal{F}_{i}\right|_{X_{i j}}$ isomorphisms such that $\varphi_{i i}=\mathrm{id}$ and $\varphi_{i j}=\varphi_{j i}^{-1}$. On the triple overlaps $X_{i j k}=\left(T_{i} \cap T_{j} \cap T_{k}\right) \times_{S} X$, the composition $\varphi_{i j} \circ \varphi_{j k} \circ \varphi_{k i}$ is an automorphism of $\left.\mathcal{F}_{i}\right|_{X_{i j k}}$. Because $\mathcal{F}_{i}$ is a simple vector bundle on 
each fibre of $q$, this composition must be a multiple of the identity. More precisely, by Lemma 2.5 there exists $\alpha_{i j k} \in \mathcal{O}\left(T_{i} \cap T_{j} \cap T_{k}\right)$, such that $\varphi_{i j} \circ \varphi_{j k} \circ \varphi_{k i}=q^{*}\left(\alpha_{i j k}\right) \cdot$ id. These functions $\alpha_{i j k}$ define a Cech cocycle $\alpha$ with values in the sheaf of abelian groups $\mathcal{O}_{T}^{*}$. Let us denote its cohomology class by $\bar{\alpha} \in H^{2}\left(\mathcal{O}_{T}^{*}\right)$.

The given sheaves $\mathcal{F}_{i}$ glue to a sheaf $\mathcal{F}$ on $X_{T}$ if and only if $\alpha_{i j k}=1$ for all $i, j, k$. Moreover, there exist line bundles $\mathcal{L}_{i}$ on $T_{i}$ such that the $\mathcal{F}_{i} \otimes q^{*} \mathcal{L}_{i}$ glue to a sheaf $\mathcal{F}$ on $X_{T}$ if and only if $\bar{\alpha}=1$ in $H^{2}\left(\mathcal{O}_{T}^{*}\right)$.

It is convenient to use the language of twisted sheaves in this situation. The sheaves $\mathcal{F}_{i}$ together with the isomorphisms $\varphi_{i j}$ define a $q^{*} \alpha$-twisted sheaf on $X_{T}$. We are going to construct an $\alpha$-twisted line bundle on $T$. The existence of it implies already $\bar{\alpha}=1$. More explicitly, we can form the tensor product of the given $q^{*} \alpha$ twisted sheaf with the pull back of the dual of this $\alpha$-twisted line bundle. This produces the desired (untwisted) sheaf $\mathcal{F}$ on $X_{T}$.

Let $\mathcal{L}_{i}=\mathbb{L}_{T_{i}}\left(\mathcal{F}_{i}\right)$ and $\psi_{i j}=\mathbb{L}_{T_{i j}}\left(\varphi_{i j}\right)$. Lemma 4.3 (a) implies that $\mathcal{L}_{i}$ is a line bundle on $T_{i}$. Using Lemma 4.3 (b) and (c) we obtain

$$
\begin{aligned}
\left.\left.\left.\psi_{i j}\right|_{T_{i j k}} \circ \psi_{j k}\right|_{T_{i j k}} \circ \psi_{k i}\right|_{T_{i j k}} & =\left.\left.\left.\mathbb{L}_{T_{i j}}\left(\varphi_{i j}\right)\right|_{T_{i j k}} \circ \mathbb{L}_{T_{j k}}\left(\varphi_{j k}\right)\right|_{T_{i j k}} \circ \mathbb{L}_{T_{k i}}\left(\varphi_{k i}\right)\right|_{T_{i j k}} \\
& =\mathbb{L}_{T_{i j k}}\left(\left.\varphi_{i j}\right|_{X_{i j k}}\right) \circ \mathbb{L}_{T_{i j k}}\left(\left.\varphi_{j k}\right|_{X_{i j k}}\right) \circ \mathbb{L}_{T_{i j k}}\left(\left.\varphi_{k i}\right|_{X_{i j k}}\right) \\
& =\mathbb{L}_{T_{i j k}}\left(\left.\varphi_{i j} \varphi_{j k} \varphi_{k i}\right|_{X_{i j k}}\right) \\
& =\mathbb{L}_{T_{i j k}}\left(q^{*}\left(\alpha_{i j k}\right) \cdot \operatorname{id}_{\left.\mathcal{F}_{i}\right|_{X_{i j k}}}\right) \\
& =\alpha_{i j k} \cdot \operatorname{id}_{\left.\mathcal{L}_{i}\right|_{T_{i j k}}} .
\end{aligned}
$$

This shows that $\left(\left\{\mathcal{L}_{i}\right\},\left\{\psi_{i j}\right\}\right)$ is an $\alpha$-twisted line bundle on $T$. This implies $\bar{\alpha}=1$ which concludes the proof.

Proof of Theorem 4.1. Because we assume $n$ and $d$ coprime, the functors $\mathbb{L}_{T}$ exist. By Proposition 4.4, the functor $\underline{\mathrm{M}}_{X / S}^{(n, d)}$ is a sheaf in the analytic topology. Theorem 6.4 and Remark 6.7 from [16] imply now the claim.

Remark 4.5. If we fix $\mathcal{F} \in \underline{\mathrm{M}}_{X / S}^{(n, d)}(T)$, we may define

$$
T_{m}=\left\{t \in T \mid H^{1}\left(\mathcal{F}(m)_{t}\right)=0\right\}
$$

for each integer $m$, i.e. $T \backslash T_{m}$ is the support of the coherent sheaf $R^{1} q_{*}(\mathcal{F}(m))$. Because the fibres $X_{t}$ are curves, $T_{m}$ is the set over which $\mathcal{F}$ is $(m+1)$-regular. The sets $T_{m}$ are open subsets of $T$ such that $T_{m} \subset T_{m+1}$ and $T=\bigcup_{m \in \mathbb{Z}} T_{m}$. Theorem 3.1 implies that there is an integer $m_{0}$, neither depending on $\mathcal{F}$ nor on $T$, such that $T_{m_{0}}=T$.

We can look at the open subfunctors $\underline{\mathrm{M}}_{X / S}^{(n, d, m)} \subset \underline{\mathrm{M}}_{X / S}^{(n, d)}$ defined by the extra condition that $H^{1}\left(\mathcal{F}(m)_{t}\right)=0$ for all $t \in T$, i.e. $R^{1} q_{*} \mathcal{F}(m)=0$. Our argument in the proof of Proposition 4.4 shows that each $\underline{\mathrm{M}}_{X / S}^{(n, d, m)}$ is a sheaf. Theorem 3.1 implies that $\underline{\mathrm{M}}_{X / S}^{(n, d, m)}=\underline{\mathrm{M}}_{X / S}^{(n, d)}$ for all $m \geq m_{0}$. 
Remark 4.6. A proof of Proposition 4.4, in which Theorem 3.1 is not used, could possibly be carried out as follows. Similar to the definition used before, we let

$$
\mathbb{L}_{T}(\mathcal{F})=(\operatorname{det}(\mathcal{A}))^{\otimes a} \otimes(\operatorname{det}(\mathcal{B}))^{\otimes b},
$$

but this time we use $\mathcal{A}=q_{*}\left(\left.\mathcal{F}\right|_{\Sigma_{T}}\right)$ and $\mathcal{B}=\mathbf{R} q_{*}(\mathcal{F})$. The key points to be verified are now that $\mathcal{A}$ is locally free (basically because the section $\Sigma$ avoids the singularities of the fibres), that $\mathcal{B}$ is a perfect complex and that $\lambda \cdot \operatorname{id}_{\mathcal{F}}$ acts as $\lambda^{\chi(\mathcal{F})} \cdot \operatorname{id}$ on $\operatorname{det}(\mathcal{B})$.

Remark 4.7. Because local freeness is an open property, Theorem 4.1 implies that the sub-functor $\underline{\mathrm{M}}_{X / S}^{(n, d)}$ of $\underline{\mathrm{M}}_{X / S}^{(n, d)}$, which consists of families of vector bundles, is representable by an open subspace $\breve{\mathrm{M}}_{X / S}^{(n, d)}$ of $\mathrm{M}_{X / S}^{(n, d)}$. In particular, under the assumptions made in the introduction, the functor $\underline{\mathrm{Pic}}_{X / S}^{d}=\underline{\mathrm{M}}_{X / S}^{(1, d)}$ is representable by a complex space $\operatorname{Pic}_{X / S}^{d}$. This functor is defined without sheafification; the elements of $\underline{\mathrm{Pic}}_{X / S}^{d}(T)$ are equivalence classes of line bundles on $X_{T}$.

Remark 4.8. If $S$ is a reduced point, $X$ an irreducible projective curve of arithmetic genus 1 , and $n>0$ and $d$ coprime integers, there is an isomorphism

$$
\text { Det : } \breve{\mathrm{M}}_{X}^{(n, d)} \longrightarrow \mathrm{Pic}_{X}^{d} \text {. }
$$

The morphism Det is determined by the condition $\operatorname{Det}^{*} \mathcal{L} \cong \operatorname{det}(\mathcal{P})$, where $\mathcal{L}$ on $\mathrm{Pic}_{X}^{d}$ and $\mathcal{P}$ on $\breve{\mathrm{M}}_{X}^{(n, d)}$ are universal sheaves. Note that, because $g=1$, for a vector bundle $V$ on $X$ we have $\operatorname{deg}(V)=\chi(V)$, so that indeed $\chi(V)=\chi(\operatorname{det}(V))$.

It was shown by Atiyah [2, Theorem 7] if $X$ is smooth and for instance in [10, Theorems 5.1.19 and 5.1.34] in the singular case, that the map Det is bijective. Because the tensor product with a line bundle of degree $d$ defines an isomorphism between $\mathrm{Pic}_{X}^{0}$ and $\mathrm{Pic}_{X}^{d}$ and because $\mathrm{Pic}_{X}^{0}$ is a group, $\mathrm{Pic}_{X}^{d}$ is smooth. This is sufficient to conclude from the bijectivity of Det that this morphism in fact is an isomorphism, see e.g. [20, Theorem 4.6.8].

Acknowledgements. Both authors would like to thank Manfred Lehn for discussing the proofs of Lemmas 2.5 and 4.2 and Norbert Hoffmann for suggesting the construction in Remark 4.6. The work of the first-named author was supported by the DFG grant Bu-1866/2-1.

\section{REFERENCES}

[1] A. Altman, S. Kleiman, Compactifying the Picard scheme, Adv. Math. 35 (1980) 50-112.

[2] M. Atiyah, Vector bundles over an elliptic curve, Proc. Lond. Math. Soc. (3) 7 (1957) 414-452.

[3] M. Atiyah, I. Macdonald, Introduction to commutative algebra, Addison-Wesley Publishing Company (1969).

[4] M. Baker, J. Csirik, An alternative approach to Serre duality for projctive varieties, http://www.csirik.net/serre-duality-long.pdf 
[5] C. Bănică, O. Stănăşilă, Algebraic Methods in the Global Theory of Complex Spaces, John Wiley (1976) $296 \mathrm{p}$.

[6] C. Bănică, M. Putinar, G. Schumacher, Variation der globalen Ext in Deformationen kompakter komplexer Räume, Math. Ann. 250 (1980) 135-155.

[7] J. Bingener, Darstellbarkeitskriterien für analytische Funktoren, Ann. Sci. Éc. Norm. Supér. (4) 13 (1980) 317-347.

[8] W. Bruns, J. Herzog, Cohen-Macaulay rings, Cambridge Studies in Advanced Mathematics 39, Cambridge: Cambridge University Press (1993).

[9] I. Burban, T. Henrich, Vector bundles on plane cubic curves and the classical Yang-Baxter equation, arXiv:1202.5738.

[10] I. Burban, B. Kreußler, Vector bundles on degenerations of elliptic curves and Yang-Baxter equations, Mem. Amer. Math. Soc. 220 (2012) no. 1035, arXiv:0708.1685.

[11] J. Dieudonné, Topics in local algebra, Lectures delivered at the University of Notre Dame. Ed. and supplemented by Mario Borelli. University of Notre Dame Press (1967) 122 p.

[12] G.-M. Greuel, On deformation of curves and a formula of Deligne, Algebraic geometry, Proc. int. Conf., La Rabida/Spain 1981, Lect. Notes Math 961 141-168 (1982).

[13] A. Grothendieck, Fondements de la géométrie algébrique, Extraits du Séminaire Bourbaki 1957-1962, Secrétariat mathématique, Paris (1962).

[14] R. Hartshorne, Algebraic geometry, Graduate Texts in Math., no. 52, Springer-Verlag (1977).

[15] D. Huybrechts, M. Lehn, The Geometry of Moduli Spaces of Sheaves. Aspects of Mathematics, E31. Friedr. Vieweg \& Sohn, Braunschweig, (1997) 269 p.

[16] S. Kosarew, C. Okonek, Global Moduli Spaces and Simple Holomorphic Bundles, Publ. RIMS Kyoto Univ. 25 (1989) 1-19.

[17] A. Norton, Analytic moduli of complex vector bundles, Indiana Univ. Math. J. 28 (1979) $365-387$.

[18] A. Polishchuk, Classical Yang-Baxter equation and the $A_{\infty}$-constraint, Adv. Math. 168 (2002), no. 1, 56-95, arXiv:math/0008156.

[19] A. Rudakov, Stability for an Abelian Category, J. Algebra 197 (1997) 231-245, arXiv:math/0312442.

[20] J. Taylor, Several Complex Variables with Connections to Algebraic Geometry and Lie Groups, Graduate Studies in Mathematics 46, AMS (2002) 507 p.

[21] A. Vistoli, Grothendieck topologies, fibered categories and descent theory, in: Fundamental Algebraic Geometry, by B. Fantechi et al., American Mathematical Society (2005) 1-104, arXiv:math/0412512.

Universität Zu KÖln, Mathematisches Institut, Weyertal 86-90, D-50931 Köln, Germany

E-mail address: burban@math.uni-koeln.de

Mary Immaculate College, South Circular Road, Limerick, Ireland

E-mail address: bernd.kreussler@mic.ul.ie 